\title{
Prediabetes can be Reversed Using Low Dose Tadalafil: Non- Classical Treatment of Diabetes as A New Medical Hypothesis
}

\author{
Ahed J Alkhatib*1, 2 \\ ${ }^{1}$ Department of Legal Medicine, Toxicology and Forensic Medicine, Jordan University of Science \& Technology, Jordan \\ ${ }^{2}$ International Mariinskaya Academy, Department of Medicine and Critical Care, Department of Philosophy, Academician Secretary of \\ Department of Sociology
}

*Corresponding author: Ahed J Alkhatib, Department of Legal Medicine, Toxicology and Forensic Medicine, Jordan University of Science \& Technology, Jordan

\begin{abstract}
Diabetes type 2 represents a global health problem, and it is expected to influence approximately 530 million people by 2030 . Usually, diabetes type 2 is developed through more than one stage. Prediabetes is a preliminary stage of diabetes before developing full episodes of diabetes.Diabetes type 2 can be preventable following appropriate lifestyles. The main objective of this study is to describe a case with liver cirrhosis and the patient was recommended for liver transplantation. Following reviewing of his profile, it was apparent that the patient had prediabetes (glucose level was $126 \mathrm{mg} / \mathrm{dl}$ ). The patient was recommended to use tadalafil $5 \mathrm{mg}$ daily to offer improvement of liver function based on previous experience. Glucose level was daily measured. It was interestingly found that glucose levels were restored to normal levels between $85-100 \mathrm{mg} / \mathrm{dl}$. Taken together, prediabetes can be treated using non-classical therapeutic approaches and full events can be prevented accordingly.
\end{abstract}

Keywords: Diabetes Type 2; Pre-Diabetes; Tadalafil; Glucose; Liver

\section{Introduction}

Type2 diabetes (T2D) can result from various reasons, it develops with time [1]. Lifestyle behaviors and environmental factors play significant roles in developing T2D [2]. Risk factors attributing to T2D include age, obesity, race, family history of T2D, abdominal adiposity and physical inactivity, and depression [1,3]. The prevalence of type 2 diabetes is dramatically increasing at international level [4].

The American Diabetes Association (ADA) defined pre-diabetes in terms of impairment in fasting glucose (5.6-6.9 mmol/L), or impairment in oral glucose tolerance test (2-h OGTT glucose 7.8$11.0 \mathrm{mmol} / \mathrm{L}$ ] [5]. Pre-diabetic can be determined by a low glucose tolerance and delayed insulin secretion [6].

Pre-diabetes may pass unnoticed because patients do not clinically exhibit specific symptoms that require medical interactions, and it needs to be investigated thoroughly because people may have various hypoglycemic states. Pre-diabetes can be fully developed into diabetes $[7,8]$.

We have recently reported a paper about induction of prediabetic model in rats. We demonstrated in this study that the use of Urtica pilluliferain restoring pre-diabetic status into normal status in terms of glucose level, lipid profile, and oxidation stress [4]. However, our accumulated experience in diabetic studies showed that development of diabetes is associated with molecular changes in different tissues such as brain [9], and liver [10]. The most common feature in our studies is the upregulation of HSP70 as a factor showing improvement in diabetic status.

Tadalafil is a member of phosphodiesterase- 5 inhibitors, and it is widely used for the treatment of erectile dysfunction due to its long acting properties [11]. The use of tadalafil has been associated with antioxidant activities in blood and tissues [12-15].[16] reported the existence of certain benefits of using daily tadalafil 
$5 \mathrm{mg}$ such as increasing some regional cerebral blood flow (CBF) as well as improving cognitive function among patients who have erectile dysfunction (ED), and with those who have mild cognitive impairment. In another study by [17], it was found that there was an influence of using a chronic tadalafil treatment in cognitive function of Alzheimer Disease (AD). We also previously found that in rat diabetic model, heat shock protein was downregulated in the white matter [18].

\section{Studycase}

A male, 32 years old, had liver cirrhosis. He was recommended to subject for liver transplantation. After reviewing his profile, he had several abnormal findings including liver function test, lactate dehydrogenase enzyme (LDH). His glucose level was estimated from $126-160 \mathrm{mg} / \mathrm{dl}$, but in most readings, his glucose level was about $130 \mathrm{mg} / \mathrm{dl}$. The patient and his family members were looking for other therapeutic options. He was recommended to use low dose tadalafil (5 mg/daily). The course was recommended for one month. During that time, the patient was required to measure fasting blood glucose daily at the morning.

\section{Results}

The main results were as the following: glucose level started declining and reached $100-93 \mathrm{mg} / \mathrm{dl}$ within the first 5 days. After one month, biochemical and hematological tests were carried out. Fasting blood sugar was $94 \mathrm{mg} / \mathrm{dl}$. Lactate dehydrogenase level dropped from 2000-1200 U/L. Liver function tests were almost within their normal reference.

\section{Discussion}

We have conducted several studies on T2D that led us to think of diabetes to be more neurological than its metabolic classical perspectives $[9,18]$. The use of low dose tadalafil has been reported to have beneficial effects in various cases of depression by working as antioxidant [11-15].

We think that detection and treatment of pre-diabetic cases is possible and feasible to prevent the development of full episode of diabetes. From accumulative experience of our studies, we think that the use of tadalafil upregulates the expression of HSP70.

\section{Conclusion}

The use of low dose tadalafil is beneficial in treating prediabetes to prevent the development of diabetes.

\section{References}

1. Ahed J Alkatib (2017) Diabetes and diabetic neuropathies are independent events: a new medical hypothesis. Ind Res J Pharm \& Sci 4(3): 1064-1067.

2. Karen A Nunley (2014) Changes in brain structure and function: underrecognized complications of type 1 diabetes. $\mathrm{PhD}$ thesis, University of Pittsburgh, Pennsylvania, USA.
3. An Pan, Nana Keum, Olivia I Okereke, Qi Sun, Mika Kivimaki, et al. (2012) Bidirectional association between depression and metabolic syndrome: A systematic review and meta-analysis of epidemiological studies. Diabetes Care 35(5): 1171-1180.

4. Kawther Faisal Amawi, Ahed J Alkhatib (2020) Urtica Pilulifera in Treating Pre-Diabetic Rat Model to Control the Blood Glucose, Lipids and Oxidative Stress. Med Arch 74(3): 168-171.

5. Katulanda GW, Katulanda P, Dematapitiya C, Dissanayake HA, Wijeratne $S$, et al. (2019) Plasma glucose in screening for diabetes and prediabetes: how much is too much? Analysis of fasting plasma glucose and oral glucose tolerance test in Sri Lankans. BMC Endocrine Disorders 19: 11.

6. Simone Renner, Christiane Fehlings, Nadja Herbach, Andreas Hofmann, Dagmar C von Waldthausen, et al. (2010) Glucose intolerance and reduced proliferation of pancreatic beta-cells in transgenic pigs with impaired glucose dependent insulinotropic polypeptide function. Diabetes 59(5): 1228-1238.

7. Kristensen SL, Preiss D, Jhund PS, Squire I, Cardoso JS, et al. (2016) Risk Related to Pre-Diabetes Mellitus and Diabetes Mellitus in Heart Failure With Reduced Ejection Fraction: Insights From Prospective Comparison of ARNI With ACEI to Determine Impact on Global Mortality and Morbidity in Heart Failure Trial. Circ Heart Fail 9(1): e002560.

8. Roberts S, Barry E, Craig D, Airoldi M, Bevan G, Greenhalgh T (2017) Preventing type 2 diabetes: systematic review of studies of costeffectiveness of lifestyle programmes and metformin, with and without screening, for pre-diabetes. BMJ Open 7(11): 1-18.

9. Ahed Jumah Alkhatib (2013) Co-expression of iNOS and HSP70 in diabetes type 1 makes a rational hypothesis to explain the diabetic neuropathy. Eur Scientific J 9(3): 145-156.

10. Kawther Faisal Amawi, Inas Saleh Al Mazari, Ali Alsarhan, Hamza Qasim Mohammad Alhamad, Ahed J Alkhatib (2019) Diabetes upregulates the expression of HSP90 and downregulates HSP70 in the liver of diabetic rats. Comp Clin Pathol 28: 473-478.

11. Coward RM, Carson CC (2008) Tadalafil in the treatment of erectile dysfunction. Ther Clin Risk Manag 4(6):1315-1330.

12. Verit A, Savas M, Ciftci H, Aksoy N, Taskin A, et al. (2010) Assessment of the acute effects of tadalafil on the cardiovascular system based on examination of serum oxidative status and paraoxonase activity in men with erectile dysfunction: a preliminary study. Int J Impot Res 22(2): 115-119.

13. Chen Y, Li XX, Lin HC, Qiu XF, Gao J, et al. (2012) The effects of long-term administration of tadalafil on STZ-induced diabetic rats with erectile dysfunction via a local antioxidative mechanism. Asian J Androl 14(4): 616-620.

14. Koka S, Das A, Salloum FN, Kukreja RC (2013) Phosphodiesterase 5 inhibitor tadalafil attenuates oxidative stress and protects against myocardial ischemia/reperfusion injury in type 2 diabetic mice. Free Radic Biol Med 60: 80-88.

15. Al Amin MM, Hasan SM, Alam T, Hasan AT, Hossain I, et al. (2014) Tadalafil enhances working memory, and reduces hippocampal oxidative stress in both young and aged mice. Eur J Pharmacol 745: 84-90.

16. Jin Bong Choi, Kang Jun Cho, Joon Chul Kim, Chung Ho Kim, Yong An Chung, et al. (2019) The Effect of Daily Low Dose Tadalafil on Cerebral Perfusion and Cognition in Patients with Erectile Dysfunction and Mild Cognitive Impairment. Clinical Psychopharmacology and Neuroscience 17(3): 432-437.

17. Carolina García Barroso, Ana Ricobaraza, María Pascual Lucas, Nora Unceta, Alberto J Rico, et al. (2013) Tadalafil crosses the blood brain barrier and reverses cognitive dysfunction in a mouse model of AD. Neuropharmacology 64: 114-123. 
18. Ahed J Alkhatib (2019) New Insights to Diabetes: Is Diabetes a Metabolic Disorder or a Neurological Disease? International Journal of Diabetes \& Metabolic Disorders 4(1): 1-2.

\section{(C) (P) This work is licensed under Creative}

To Submit Your Article Click Here:

Submit Article

DOI: $10.32474 / A D 0.2020 .02 .000150$

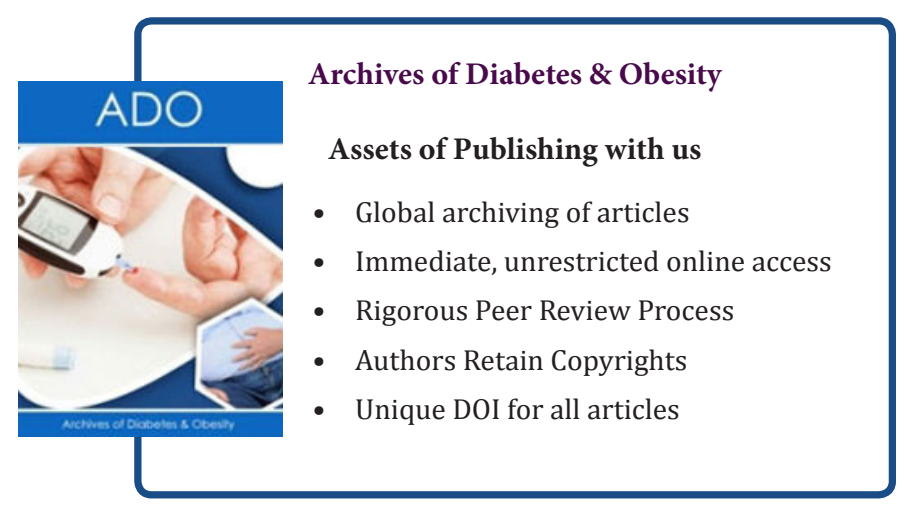

